J. Astron. Space Sci. 27(1), 1-10 (2010)

\title{
Titius-Bode's Relation and Distribution of Exoplanets
}

\section{Heon-Young Chang}

\author{
Department of Astronomy and Atmospheric Sciences, Kyungpook National University \\ 1370 Sankyuk-dong, Buk-gu, Daegu 702-701, Korea \\ email: hyc@knu.ac.kr
}

(Received November 24, 2009; Accepted January 13, 2010)

\begin{abstract}
The distance distribution in our planetary system has been a controversial matter. Two kinds of important issues on Titius-Bode's relation have been discussed up to now: one is if there is a simple mathematical relation between distances of natural bodies orbiting a central body, and the other is if there is any physical basis for such a relation. We have examined, by applying it to exo-planetary systems, whether Titius-Bode's relation is exclusively applicable to our solar system. We study, with the $\chi^{2}$ test, the distribution of period ratios of two planets in multiple planet systems by comparing it with that derived from not only Titius-Bode's relation but also other forms of it. The $\chi^{2}$ value between the distribution of the orbital period derived from Titius-Bode's relation and that observed in our Solar system is $12.28(\mathrm{dof}=18)$ with high probability, i.e., $83.3 \%$. The value of $\chi^{2}$ and probability resulted from Titius-Bode's relation and observed exo-planetary systems are $21.38(\mathrm{dof}=26)$ and $72.2 \%$, respectively. Modified forms we adopted seem also to agree with the planetary system as favorably as Titius-Bode's relation does. As a result, one cannot rule out the possibility that the distribution of the ratio of orbiting periods in multiple planet systems is consistent with that derived from Titius-Bode's relation. Having speculated Titius-Bode's relation could be valid in exo-planetary systems, we tentatively conclude it is unlikely that Titius-Bode's relation explains the distance distribution in our planetary system due to chance. Finally, we point out implications of our finding.
\end{abstract}

Keywords: celestial mechanics - solar system - general

\section{Introduction}

Johann Daniel Titius von Wittenberg first attempted in 1766 to representat planetary distances with an empirical relation giving the semi-major axis $a_{n}$ of a planet in AU as

$$
a_{n}=0.4+\left(0.3 \times 2^{n}\right)
$$

where $n$ is given by $-\infty$ for Mercury, 0 for Venus, 1 for Earth and so on. With the recognition by Johann Elert Bode in 1782 that Titius' relation predicted the semi-major axis of Uranus and the 'successful' discovery of Ceres by Giuseppe Piazzi in 1801 with the semi-major axis predicted by Titius-Bode's relation, it has been seemingly adopted as a 'law' (e.g., for further discussions see Nieto 1972). Other expressions of Titius-Bode's relation have been proposed by several authors 
(Blagg 1913, Richardson 1945, Dermott 1968, Prentice 1977, Isaacman \& Sagan 1977, Rawal 1978, 1984, 1986, 1989, Basano \& Hughes 1979, Neuhäuser \& Feitzinger 1986, Ragnarsson 1995, Ortiz et al. 2007). Interestingly enough, relations similar to eq. (1) can be found for the satellite systems of giant planets in the Solar system as well (Nieto 1970, Pletser 1986, Rawal 1984, 1986, 1989, Li, Zhang, \& Li 1995). It should be noted that there are also 'missing holes' to the satellite systems, corresponding to the asteroidal belt in the planetary system, which is indeed filled by rings and small satellites (e.g., Stone \& Miner 1986).

Titius-Bode's relation embarrassingly breaks down for Neptune and objects farther than that, while it gives neat results for the eight first planets, including the asteroids. This is where people begin to doubt Titius-Bode's relation. Moreover, most of researchers do believe that there is no physical basis to explain the apparent simple mathematical relation between distances of natural bodies revolving around a central body, though several theories of the solar system formation have attempted to account for these distance relations (Dermott 1972, 1973, Dobó 1981, Louise 1982, Llibre \& Pinõl 1987, Patton 1988, Graner \& Dubrulle 1994a,b, Li, Zhang, \& Li 1995, Nottale, Schumacher, \& Gay 1997, Ortiz et al. 2007). Attempts from other directions to explain the origin of the relation have been also made. For instance, some authors have sought to find a solution among random processes (e.g., Lecar 1973, Pletser 1988, Hayes \& Tremaine 1998, Neslušan 2004).

The question of whether the observed patterns have some physical reasons or are due to chance may be addressed using a Monte Carlo approach. Lynch (2003), however, finds that the estimated probability of chance occurrence depends sensitively on restrictions imposed on the population of orbits. Another strategy to pursue is testing whether Titius-Bode's relation is exclusively applicable to our own Solar system. If it is not the case, it would indirectly demonstrate that Titius-Bode's relation is more than pure numerological speculations and to be understood. In fact, Titius-Bode's relation has been recently tried to exo-planetary systems. For instance, Vahia, Mahajani, \& Rao (2003) have investigated the distribution of extrasolar system planets to search for periodicities in their distribution around their parent star. More recently, Poveda \& Lara (2008) have investigated if the exo-planetary system, 55 Cancri (= HD 75732), fits some form of Titius-Bode's relation.

In this paper, we study the distribution of period ratios of two planets in multiple planet systems by comparing it with that derived from not only Titius-Bode's relation but also other forms of it. According to Kepler's 3rd law and to a generalized Titius-Bode's relation, i.e., $a_{n} \propto \beta^{n}$, the ratio of the orbital periods of two planets in an exo-planetary system is given as $P_{n} / P_{m}=$ $\left(a_{n}^{3 / 2}\right) /\left(a_{m}^{3 / 2}\right)=\left(\beta^{3 / 2}\right)^{n-m}$. What this means is that one may check Titius-Bode's relation in terms of the period which is directly measured by observations, instead of the semi-major axis which is secondary information deduced from observations with assumptions, e.g., on mass. As long as exo-planetary systems obey Titius-Bode's relation, $P_{n} / P_{m}$ computed with randomly selected pairs should satisfy a specific distribution function. Hence, by comparing the distribution of the ratio of periods of two planets in multiple planet systems with that derived from Titius-Bode's relation one may attempt to examine if exo-planetary systems share the same form. Instead of investigating an individual exo-planetary system one by one, we study an ensemble collectively to increase the statistical significance by assuming that the ensemble of the distributions from exo-planetary systems is approximately equivalent to the distirbution deduced by numerous random draws from a single planetary system. Taking a ratio of the orbiting period makes it feasible since the ratio is independent of mass of the planetary system. Implications are to be discussed below.

This paper begins with descriptions of the distribution of the ratio we analyze and data of exoplanetary systems in section 2 . We present results obtained with $\chi^{2}$ test in section 3 . Finally, we discuss and conclude in section 4. 

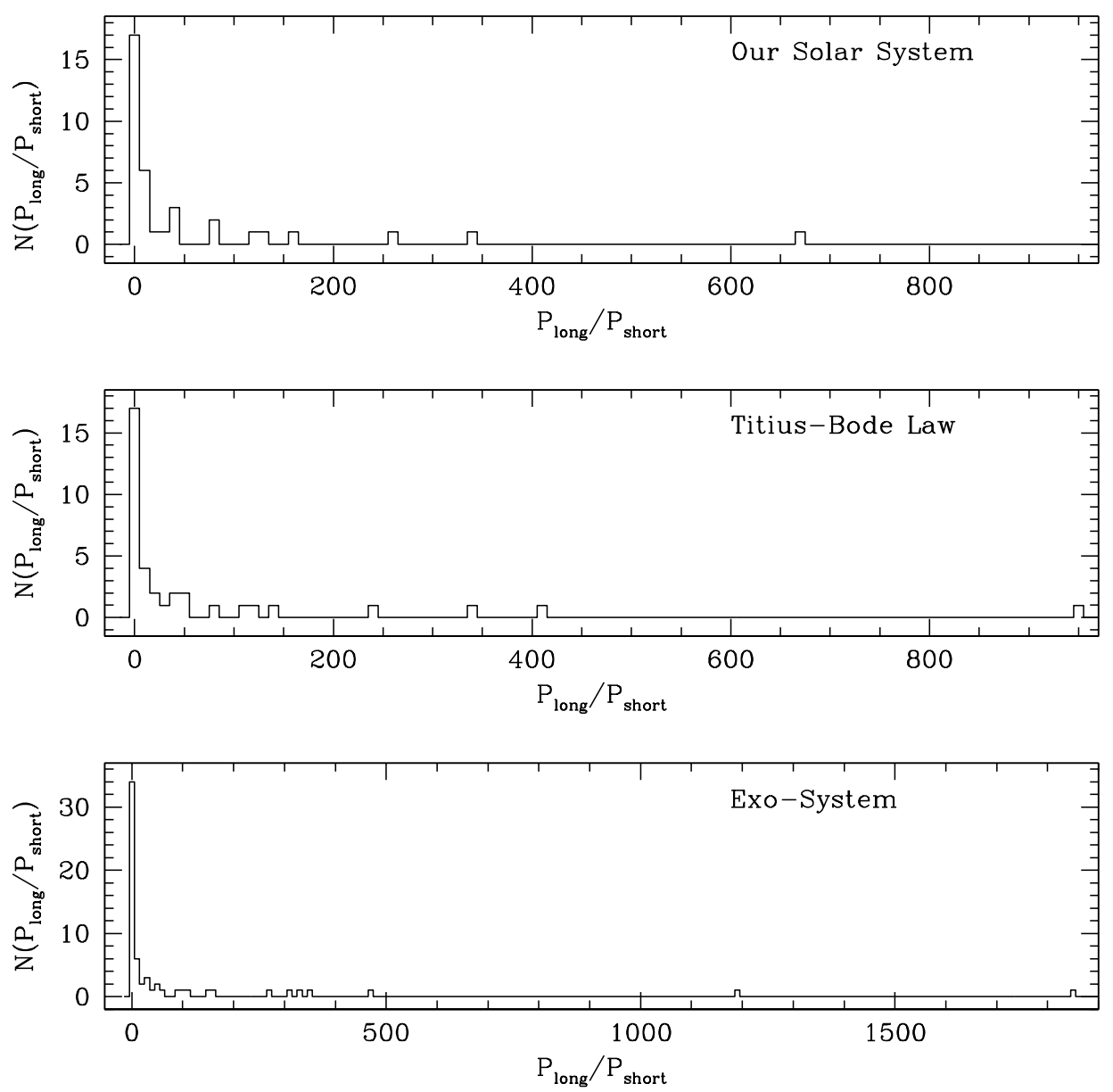

Figure 1. Distribution of the period ratio of two planets. In the top panel, the distribution from our solar system is shown. In the middle panel, the distribution of the ratio of calculated periods of two planets from Mercury to Neptune by Titius-Bode's relation is shown. In the bottom panel, we show the distribution from 31 exo-planetary systems.

\section{Distribution of Ratio of Orbital Periods}

In Figure 1, we show the distribution of period ratios of two planets. In the top panel, the distribution of the ratio of observed periods of two planets in our solar system is shown. To obtain the ratio we randomly choose two different planets and take the ratio such that the value is always greater than unity. It should be noted that we do not consider Pluto (one of large known bodies in the Kuiper-Edgeworth belt) as a proper planet. On the other hand, it appears reasonable to include Ceres as a representative of the putative former planet. Both in the classical Titius-Bode's relation 


\section{Chang}

Table 1. Multiple planet systems adopted in the present analysis. Data of planets and host stars are taken from the Extrasolar Planets Encyclopedia.

\begin{tabular}{|c|c|c|c|c|c|c|}
\hline \multirow[t]{2}{*}{ No. } & \multirow[t]{2}{*}{ Name } & \multicolumn{3}{|c|}{ Planet } & \multicolumn{2}{|c|}{ Host Star } \\
\hline & & $\operatorname{Mass}\left(M_{\mathrm{Jup}}\right)$ & Period(day) & Semi-axis(AU) & Spec. Type & St. Mass $\left(M_{\odot}\right)$ \\
\hline \multirow[t]{2}{*}{1} & 47 Uma b & 2.6 & 1083.2 & 2.11 & G0V & 1.03 \\
\hline & 47 Uma c & 0.46 & 2190 & 3.39 & & \\
\hline \multirow[t]{5}{*}{2} & $55 \mathrm{Cnc} b$ & 0.824 & 14.65162 & 0.115 & G8 V & 1.03 \\
\hline & $55 \mathrm{Cnc} c$ & 0.169 & 44.3446 & 0.24 & & \\
\hline & $55 \mathrm{Cnc} \mathrm{d}$ & 3.835 & 5218 & 5.77 & & \\
\hline & $55 \mathrm{Cnc}$ e & 0.034 & 2.81705 & 0.038 & & \\
\hline & $55 \mathrm{Cnc} \mathrm{f}$ & 0.144 & 260 & 0.781 & & \\
\hline \multirow[t]{3}{*}{3} & Gl 581 b & 0.0492 & 5.3683 & 0.041 & M3 & 0.31 \\
\hline & G1 $581 \mathrm{c}$ & 0.0158 & 12.932 & 0.073 & & \\
\hline & Gl $581 \mathrm{~d}$ & 0.0243 & 83.6 & 0.25 & & \\
\hline \multirow[t]{3}{*}{4} & Gliese 876 b & 1.935 & 60.94 & 0.20783 & M4 V & 0.32 \\
\hline & Gliese $876 c$ & 0.56 & 30.1 & 0.13 & & \\
\hline & Gliese $876 \mathrm{~d}$ & 0.018 & 1.93776 & 0.0208067 & & \\
\hline \multirow[t]{2}{*}{5} & HD 102272 b & 5.9 & 127.58 & 0.614 & K0 & 1.9 \\
\hline & HD $102272 \mathrm{c}$ & 2.6 & 520 & 1.57 & & \\
\hline \multirow[t]{2}{*}{6} & HD 108874 b & 1.36 & 395.4 & 1.051 & G5 & 1 \\
\hline & HD $108874 \mathrm{c}$ & 1.018 & 1605.8 & 2.68 & & \\
\hline \multirow[t]{2}{*}{7} & HD 11964 b & 0.11 & 37.82 & 0.229 & G5 & 1.125 \\
\hline & HD $11964 \mathrm{c}$ & 0.7 & 1940 & 3.167 & & \\
\hline \multirow[t]{2}{*}{8} & HD $12661 \mathrm{~b}$ & 2.3 & 263.6 & 0.83 & G6 V & 1.07 \\
\hline & HD $12661 \mathrm{c}$ & 1.57 & 1444.5 & 2.56 & & \\
\hline \multirow[t]{2}{*}{9} & HD $128311 b$ & 2.18 & 448.6 & 1.099 & K0 & 0.8 \\
\hline & HD $128311 \mathrm{c}$ & 3.21 & 919 & 1.76 & & \\
\hline \multirow[t]{2}{*}{10} & HD $155358 \mathrm{~b}$ & 0.89 & 195 & 0.628 & G0 & 0.87 \\
\hline & HD $155358 \mathrm{c}$ & 0.504 & 530.3 & 1.224 & & \\
\hline \multirow[t]{4}{*}{11} & HD $160691 \mathrm{~b}$ & 1.67 & 654.5 & 1.5 & G3 IV-V & 1.08 \\
\hline & HD $160691 \mathrm{c}$ & 3.1 & 2986 & 4.17 & & \\
\hline & HD $160691 \mathrm{~d}$ & 0.044 & 9.55 & 0.09 & & \\
\hline & HD $160691 \mathrm{e}$ & 0.5219 & 310.55 & 0.921 & & \\
\hline \multirow[t]{2}{*}{12} & HD $168443 b$ & 8.02 & 58.11289 & 0.3 & G5 & 1.06 \\
\hline & HD $168443 \mathrm{c}$ & 18.1 & 1765.8 & 2.91 & & \\
\hline \multirow[t]{2}{*}{13} & HD $169830 b$ & 2.88 & 225.62 & 0.81 & F8 V & 1.4 \\
\hline & HD $169830 \mathrm{c}$ & 4.04 & 2102 & 3.6 & & \\
\hline \multirow[t]{2}{*}{14} & HD 181433 b & 0.0238 & 9.37 & - & K3III-IV & - \\
\hline & HD $181433 \mathrm{c}$ & 0.72 & 1024 & - & & \\
\hline 15 & HD $187123 b$ & 0.52 & 3.097 & 0.042 & G5 & 1.06 \\
\hline & HD $187123 \mathrm{c}$ & 1.95 & 3700 & 4.8 & & \\
\hline 16 & HD $190360 \mathrm{~b}$ & 1.502 & 2891 & 3.92 & G6 IV & 1.04 \\
\hline & HD $190360 \mathrm{c}$ & 0.057 & 17.1 & 0.128 & & \\
\hline 17 & HD 202206 b & 17.4 & 255.87 & 0.83 & G6 V & 1.13 \\
\hline & HD $202206 \mathrm{c}$ & 2.44 & 1383.4 & 2.55 & & \\
\hline 18 & HD $217107 \mathrm{~b}$ & 1.33 & 7.12689 & 0.073 & G8 IV & 1.02 \\
\hline & HD $217107 \mathrm{c}$ & 2.5 & 3352 & 4.41 & & \\
\hline 19 & HD 37124 b & 0.61 & 154.46 & 0.53 & G4 V & 0.91 \\
\hline & HD 37124 c & 0.683 & 2295 & 3.19 & & \\
\hline & HD $37124 \mathrm{~d}$ & 0.6 & 843.6 & 1.64 & & \\
\hline 20 & HD $38529 b$ & 0.78 & 14.309 & 0.129 & G4 IV & 1.39 \\
\hline & HD $38529 c$ & 12.7 & 2174.3 & 3.68 & & \\
\hline 21 & HD $40307 \mathrm{~b}$ & 0.0132 & 4.3115 & 0.047 & $\mathrm{~K} 2.5 \mathrm{~V}$ & - \\
\hline & HD $40307 \mathrm{c}$ & 0.0216 & 9.62 & 0.081 & & \\
\hline & HD $40307 \mathrm{~d}$ & 0.0288 & 20.46 & 0.134 & & \\
\hline 22 & HD $47186 b$ & 0.0717 & 4.08 & - & G6V & - \\
\hline & HD $47186 \mathrm{c}$ & 0.35 & 1354 & - & & \\
\hline
\end{tabular}


Table 1. Continued

\begin{tabular}{|c|c|c|c|c|c|c|}
\hline \multirow[t]{2}{*}{ No. } & \multirow[t]{2}{*}{ Name } & \multicolumn{3}{|c|}{ Planet } & \multicolumn{2}{|c|}{ Host Star } \\
\hline & & $\operatorname{Mass}\left(M_{\text {Jup }}\right)$ & Period(day) & Semi-axis(AU) & Spec. Type & St. Mass $\left(M_{\odot}\right)$ \\
\hline \multirow[t]{2}{*}{23} & HD 47536 b & 5 & 430 & - & K1 III & 0.94 \\
\hline & HD $47536 \mathrm{c}$ & 7 & 2500 & - & & \\
\hline \multirow[t]{3}{*}{24} & HD $69830 \mathrm{~b}$ & 0.033 & 8.667 & 0.0785 & KOV & 0.86 \\
\hline & HD 69830 c & 0.038 & 31.56 & 0.186 & & \\
\hline & HD $69830 \mathrm{~d}$ & 0.058 & 197 & 0.63 & & \\
\hline \multirow[t]{2}{*}{25} & HD $73526 b$ & 2.9 & 188.3 & 0.66 & G6 V & 1.02 \\
\hline & HD $73526 \mathrm{c}$ & 2.5 & 377.8 & 1.05 & & \\
\hline \multirow[t]{3}{*}{26} & HD $74156 b$ & 1.88 & 51.65 & 0.294 & G0 & 1.24 \\
\hline & HD 74156 c & 8.03 & 2476 & 3.85 & & \\
\hline & HD $74156 \mathrm{~d}$ & 0.396 & 336.6 & 1.01 & & \\
\hline \multirow[t]{2}{*}{27} & HD 82943 b & 1.75 & 441.2 & 1.19 & G0 & 1.18 \\
\hline & HD 82943 c & 2.01 & 219 & 0.746 & & \\
\hline \multirow[t]{2}{*}{28} & HIP $14810 b$ & 3.84 & 6.6742 & 0.0692 & G5 & 0.99 \\
\hline & HIP $14810 \mathrm{c}$ & 0.76 & 95.2914 & 0.407 & & \\
\hline \multirow[t]{2}{*}{29} & OGLE-06-109L b & 0.71 & 1825 & 2.3 & - & 0.5 \\
\hline & OGLE-06-109L c & 0.27 & 5100 & 4.6 & & \\
\hline \multirow[t]{3}{*}{30} & PSR 1257+12 b & $7.00 \mathrm{E}-05$ & 25.262 & 0.19 & - & - \\
\hline & PSR $1257+12 \mathrm{c}$ & 0.013 & 66.5419 & 0.36 & & \\
\hline & PSR $1257+12 d$ & 0.012 & 98.2114 & 0.46 & & \\
\hline \multirow[t]{3}{*}{31} & Ups And b & 0.69 & 4.61708 & 0.059 & F8 V & 1.27 \\
\hline & Ups And c & 1.98 & 241.52 & 0.83 & & \\
\hline & Ups And d & 3.95 & 1274.6 & 2.51 & & \\
\hline
\end{tabular}

and in almost all of its modifications, the mean asteroid-belt distance is included in the regular planetary distances. Thus, an exception of the Pluto to the observed distribution alone is allowed. The middle panel shows the distribution of the ratio of calculated periods of two planets from Mercury to Neptune using Titius-Bode's relation. Again, the distribution is obtained by randomly chosen pairs of planets. In the bottom panel, we show the distribution of the ratio of observed periods of two planets in multiple exo-planetary systems listed in the Extrasolar Planets Encyclopedia ${ }^{1}$, in which discovered exoplanets are constantly updated by Jean Schneider. We necessarily consider multiple planet systems only, in which more than one planet are found to be orbiting around a host star so that the ratio of periods can be obtained. For exo-planetary systems where more than two planets are detected, we consider all the possible combinations in calculating the ratio. According to the Extrasolar Planets Encyclopedia, there are 307 planets detected in 263 exo-planetary systems as of July in 2008. Among these 31 planet systems are multiple. In Table 1, we list multiple planet systems used in our present analysis. Note that except PSR 1257+12 detected by timing technique and OGLE-06-109L by microlensing observations, 29 systems are detected by the radial velocity method.

\section{Results}

We perform a statistical test to check if the observed ratio distributions are consistent with that derived from Titius-Bode's relation, by calculating $\chi^{2}$ values between two binned distributions resulting from the ratio of the orbital period. In Table 2, we show the $\chi^{2}$ value with the degrees of

\footnotetext{
${ }^{1}$ http://exoplanet.eu/catalog-all.php
} 


\section{Chang}

Table 2. Results of the $\chi^{2}$ test. The first and second columns indicate the data set from which the distribution is derived. Values of $\chi^{2}$, the degrees of freedom, dof, and the probability, P, indicating two data sets are drawn from the same distribution function are listed in the third, fourth, and fifth columns, respectively.

\begin{tabular}{ccrcc}
\hline \hline & & $\chi^{2}$ & dof & P \\
\hline Titus-Bode & Solar System & 12.28 & 18 & 83.3 \\
& Exo-System & 21.38 & 26 & 72.2 \\
Titus-Bode(Neptune) & Solar System & 6.15 & 14 & 96.2 \\
& Exo-System & 16.85 & 23 & 81.7 \\
\hline
\end{tabular}

Table 3. Similar to Table 2, except that the adopted bin size is 5 instead of 10 .

\begin{tabular}{ccccc}
\hline \hline & & $\chi^{2}$ & dof & P \\
\hline Titus-Bode & Solar System & 21.8 & 26 & 69.9 \\
& Exo-System & 22.2 & 29 & 81.1 \\
Titus-Bode(Neptune) & Solar System & 15.2 & 22 & 85.4 \\
& Exo-System & 17.8 & 26 & 88.5 \\
\hline
\end{tabular}

Table 4. Similar to Table 2, except that the adopted bin size is 20 instead of 10.

\begin{tabular}{ccrrc}
\hline \hline & & $\chi^{2}$ & dof & $\mathrm{P}$ \\
\hline Titus-Bode & Solar System & 9.1 & 14 & 82.5 \\
& Exo-System & 13.3 & 19 & 82.1 \\
Titus-Bode(Neptune) & Solar System & 4.1 & 10 & 94.2 \\
& Exo-System & 8.7 & 16 & 92.0 \\
\hline
\end{tabular}

freedom, dof, and probability that two data sets are drawn from the same distribution function. The size of bins is fixed to the same for all the cases throughout the analysis, i.e., 10. In the first row, results of the $\chi^{2}$ test between the distribution of the orbital period derived from Titius-Bode's relation and that observed in our Solar system are shown. The $\chi^{2}$ value is 12.28 with high probability, i.e., $83.3 \%$. In the second row, the value of $\chi^{2}$ and probability resulted from Titius-Bode's relation and observed exo-planetary systems are shown. The $\chi^{2}$ value is 21.38 . The probability is $72.2 \%$. The probability is somewhat low, but not small enough to conclude that they are significantly different distributions. Hence, we tentatively conclude that the traditional Titius-Bode's relation can be applied to exo-planetary systems. For comparison, in the third and fourth rows, we show results from Titius-Bode's relation excluding Neptune are shown. As one may expect, the distribution derived from Titius-Bode's relation rounded-off at Uranus should agree more satisfactorily with the distribution observed in our Solar system. Test results show that distributions are more consistent not only in the Solar system case but also in exo-planetary system case. We repeat same calculations with various bin sizes to see how robust our results are. Results of the $\chi^{2}$ test with other bin sizes also show they are comparable with reasonably high probability, i.e., higher than $\sim 80 \%$, except the case of the Titius-Bode's relation and the our solar system with Neptune. Two example cases of results are shown in Tables 3 and 4.

As we mentioned earlier, there are various versions of Titius-Bode's relation. We also perform 

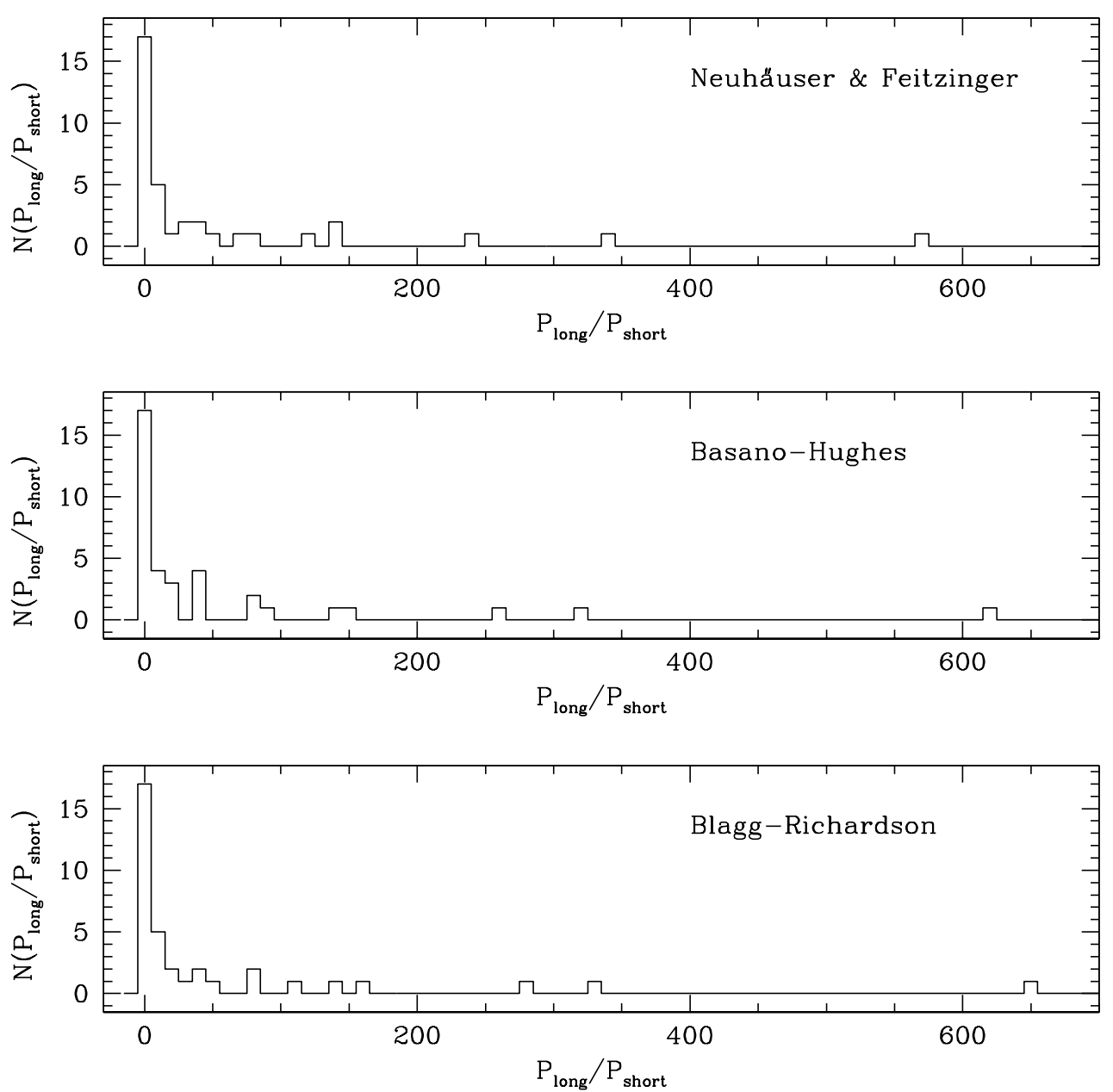

Figure 2. Distribution of the period ratio of two planets derived from various forms of Titius-Bode's relation. The name of various formulae are indicated in the upper right corner of the panel. Top panel is derived from Neuhäuser \& Feitzinger (1986), middle panel is from Basano \& Hughes (1979), and bottom panel is from Blagg (1913) and Richardson (1945).

$\chi^{2}$ tests with those modified forms. In Figure 2, we show distributions of the calculated period ratio of two planets using other forms of Titius-Bode's relation. Top panel is derived from Neuhäuser \& Feitzinger (1986), middle panel is from Basano \& Hughes (1979), and bottom panel is from Blagg (1913) and Richardson (1945). Distance formulae are designated in the upper right corner of the panel. We include Ceres but omit Neptune and Pluto, as discussed in the Titius-Bode case. Results of the $\chi^{2}$ test are summarized in Table 5. Modified forms we adopted in this particular example in general agree with the planetary system as favorably as Titius-Bode's relation does. The derived 
Table 5. Similar to Table 2, except that various forms of Titius-Bode's relations are tested as indicated. Results are from those excluding Neptune. Neuhäuser-Fritzinger's law is given as $a_{n}=a+b \times 2^{n}$, where $a$ and $b$ are given as a table determined with planets' eccentricities, $n$ is given by $-\infty, 0,1$, and so on. Basano-Hughes's law is given as $a_{n}=0.285 \times 1.523^{n}$, where $n$ runs from unity by one. Blagg-Richardson's law is given as $a_{n}=A \times 1.728^{n} \times\{B+f(\alpha+n \beta)\}$, where constants are determined empirically with a predetermined function, $n$ is given by $-2,-1,0,1$, and so on. See the text for references of the formulae.

\begin{tabular}{ccccc}
\hline \hline & & $\chi^{2}$ & dof & $\mathrm{P}$ \\
\hline Neuhäuser-Fritzinger & Solar System & 19.68 & 13 & 83.7 \\
& Exo-System & 21.93 & 22 & 62.1 \\
Basano-Hughes & Solar System & 12.02 & 14 & 74.3 \\
& Exo-System & 23.67 & 20 & 37.7 \\
Blagg-Richardson & Solar System & 16.83 & 20 & 77.4 \\
& Exo-System & 15.19 & 20 & 79.3 \\
\hline
\end{tabular}

distributions are consistent with observed one in the Solar system with probability higher than $\sim$ $80 \%$, except the distance formula of Basano \& Hughes (1979). Results of the $\chi^{2}$ test with the relation of Basano \& Hughes (1979) become even worse in the case of exo-planetary system. It may reflect drawbacks of the relation (e.g., for further discussion on comparison of distance relations see Neuhäuser \& Feitzinger 1986).

\section{Discussion and Conclusion}

We have examined whether Titius-Bode's relation is also applicable to exo-planetary systems. For this purpose, we statistically study the distribution of observed orbiting period ratios of two planets in multiple exo-planetary systems by comparing it with that derived from various forms of Titius-Bode's relation. As a result of the $\chi^{2}$ test we have carried out, one cannot rule out the possibility that the distribution of the ratio of orbiting periods in multiple planet systems is consistent with that derived from Titius-Bode's relation. Having speculated Titius-Bode's relation could be valid in exo-planetary systems, we tentatively conclude it is unlikely that Titius-Bode's relation explains the distance distribution in our planetary system due to chance. It is, however, fair to point out a possibility that our finding is biased by the selection effect. In other words, most of extra-solar planets are detected by the radial velocity method which is sensitive to close and massive planets. One may think that this might have suppressed the distribution of period ratios at large $\mathrm{P}_{\text {long }} / \mathrm{P}_{\text {short }}$, e.g., in Figure 1, since farther planets might be found less than would be. Or, at the same time, one may also think this might have enhanced the distribution of period ratios at large $\mathrm{P}_{\text {long }} / \mathrm{P}_{\text {short }}$ since closer planets might be found more than would be. The assumption we take in this analysis that the distribution of an ensemble from exo-planetary systems is approximately equivalent to that deduced by numerous random selections from a single planetary system should be resolved when large numbers of planets be found around many stars. We also admit that one may suggest that our conclusion is inconclusive in the sense that rigorous statistical tests should be carried out in terms of properties of host stars or parameters generating sub-samples.

If Titius-Bode's relation of the solar system are more than pure numerological speculations, our finding has following implications. First one is on formation and evolution of the planetary system. The idea of planetary migration is prevalent in explaining hot Jupiters. Knowledge on the migration, such as, how to work, is found in the planetary disk dynamics. However, boundary conditions, for 
instance, where to start and/or to stop, is not coming from the first principle. We have no solid knowledge on the possible role of massive Jupiter-like planets in shaping up the distribution of planets. To this end, Titius-Bode's relation may have some constraints. Another implication to be pointed out is on the extrasolar planet search. It seems that all the planetary systems own more than one planet. Once a planet is found there is a chance to find another. Hence, it may help to discover new planets by paying special attention to periodic signals in the radial velocities at values close to the periods prediceted by Titius-Bode's relation.

Acknowledgments: This work was supported by Basic Science Research Program through the National Research Foundation of Korea (NRF) funded by the Ministry of Education, Science and Technology (2009-0071263).

\section{References}

Basano, L. \& Hughes, D. W. 1979, Il Nuovo Cimento, Vol.2C, 505

Blagg, M. A. 1913, MNRAS, 73, 414

Dermott, S. F. 1968, MNRAS, 141, 363

Dermott, S. F. 1972, in On the Origin of the Solar System-Nice Symposium, ed. H. Reeves (Paris: CNRS), p.320

Dermott, S. F. 1973, Nature, 244, 18

Dobó, A. 1981, Astron. Nachr., Bd. 302, H.2

Graner, F. \& Dubrulle, B. 1994a, A\&A, 282, 262

Graner, F. \& Dubrulle, B. 1994b, A\&A, 282, 269

Hayes, W. \& Tremaine, S. 1998, Icarus, 135, 549

Isaacman, R. \& Sagan, C. 1977, Icarus, 31, 510

Lecar, M. 1973, Nature, 242, 318

Li, X. Q., Zhang, H., \& Li, Q. B. 1995, A\&A, 304, 617

Llibre, J., \& Pinõl, C. 1987, AJ, 93, 1272

Louise, R. 1982, M\&P, 26, 93

Lynch, P. 2003, MNRAS, 341, 1174

Neslušan, L. 2004, MNRAS, 351, 133

Neuhäuser, R. \& Feitzinger, J. V. 1986, A\&A, 170, 174

Nieto, M. M. 1970, A\&A, 8, 105

Nieto, M. M. 1972, The Titius-Bode Law of Planetary Distances: Its History and Theory (Oxford: Pergamon Press)

Nottale, L., Schumacher, G., \& Gay, J. 1997, A\&A, 322, 1018

Ortiz, J. L., Moreno, F., Molina, A., Sanz, P. S., \& Gutierrez, P. J. 2007, MNRAS, 379, 1222

Patton, J. M. 1988, CeMec, 44, 365

Pletser, V. 1986, Earth, Moon, and Planets, 36, 209

Pletser, V. 1988, Earth, Moon, and Planets, 42, 1

Poveda, A. \& Lara, P. 2008, astro-ph/0803.2240

Prentice, A. J. R. 1997, in The Origin of the Solar System, ed. S. F. Dermott (New York: Wiley)

Ragnarsson, S.-I. 1995, A\&A, 301, 609

Rawal, J. J. 1978, BASI, 6, 92

Rawal, J. J. 1984, Earth, Moon, and Planets, 31, 175

Rawal, J. J. 1986, Earth, Moon, and Planets, 34, 93

Rawal, J. J. 1989, Earth, Moon, and Planets, 44, 265 


\section{Chang}

Richardson, D. E. 1945, Astron., 53, 14

Stone, E. C. \& Miner, E. D. 1986, Science, 233, 39

Vahia, M. N., Mahajani, P., \& Rao, A. R. 2003, BASI, 31, 37 